\title{
Improving Vietnamese Word Segmentation and POS Tagging using MEM with Various Kinds of Resources
}

\author{
Oanh Thi Tran ${ }^{\dagger}$, Cuong Anh $\mathrm{Le}^{\dagger}$ and Thuy Quang $\mathrm{Ha}^{\dagger}$
}

\begin{abstract}
Word segmentation and POS tagging are two important problems included in many NLP tasks. They, however, have not drawn much attention of Vietnamese researchers all over the world. In this paper, we focus on the integration of advantages from several resourses to improve the accuracy of Vietnamese word segmentation as well as POS tagging task. For word segmentation, we propose a solution in which we try to utilize multiple knowledge resources including dictionary-based model, N-gram model, and named entity recognition model and then integrate them into a Maximum Entropy model. The result of experiments on a public corpus has shown its effectiveness in comparison with the best current models. We got 95.30\% F1 measure. For POS tagging, motivated from Chinese research and Vietnamese characteristics, we present a new kind of features based on the idea of word composition. We call it morpheme-based features. Our experiments based on two POS-tagged corpora showed that morpheme-based features always give promising results. In the best case, we got $\mathbf{8 9 . 6 4 \%}$ precision on a Vietnamese POS-tagged corpus when using Maximum Entropy model.
\end{abstract}

Key Words: word segmentation, Natural language processing (NLP), dictionary-based model, Named Entity model, N-gram model, morpheme-based feature, word-based feature, POS tagging

\section{Introduction}

Word segmentation is the process of splitting a given sentence into separated words. This is the first step we have to solve before reaching further applications like part of speech tagging, syntax parsing, and document understanding. Vietnamese has specific characteristics which make Vietnamese word segmentation difficult to carry out. It is an isolated, non-inflection language; its characters are based on Latin alphabet. Like other languages such as Chinese, Japanese, and Korean, each Vietnamese word is not separated by white spaces. In fact, one word may consist of one or more morphemes and morphemes are separated by white spaces.

Some approaches to Vietnamese word segmentation have been proposed. In general, they can be divided into two main approaches: dictionary-based approach and statistics-based approach. The former is widely known with two typical methods, namely Longest Matching and

\footnotetext{
$\dagger$ College of Technology, Vietnam National University Hanoi
} 
Maximum Matching. Most of initial works in word segmentation are dictionary-based methods (Poowarawan 1986) (Wong and Chan 1996). Although this approach is simple, it is not highly effective because it cannot handle ambiguous cases and cannot discover new words. The later is used more popularly in word segmentation. Researchers have already used statistic methods to segment Chinese words (Gao 2005) (Maosong 1998), Thai words (Mekanavin et al. 1997), as well as Vietnamese words (Cam-Tu Nguyen 2007) (Dien and Thuy 2006) (Ha 2003), etc. Some of them utilized dictionary and named entities information to extract features. However, they were not interested in discovering new words (not only named entities but also factoid forms).

With regard to Part-of-speech tagging, this is the process of assigning a part-of-speech tag to each word in a sentence according to its context. Many machine learning methods have been applied for POS tagging such as Maximum entropy model for English POS tagger (Ratnaparkhi 1997), a method that performs joint decoding of separately trained CRF models for Chinese word segmentation and POS tagging task (Shi and Wang 2007) and etc... Those methods got high performance when estimated on golden corpora such as Wall Street Journal corpus using Penn Tree Bank tag sets and Penn Chinese Treebank. So far, most studies are focused on common languages such as English, Japanese and Chinese. For other languages like language of India, Thailand, Russian, or Vietnam, POS tagging is still a difficult challenge. Methods and tools which are successfully built for English usually give low results when applying for these languages (Hasan 2007). Therefore, demands for each language are either inheriting, modifying, and taking full advantages of these available methods or proposing new methods that are efficient for these language characteristics.

In this paper, we propose a method to integrate various knowledge resources with several models into a unified framework. The knowledge extracted from different resources including dictionary, N-gram, named entities, as well as context morphemes will be investigated. Among them we realized that N-gram information, which has not been used in previous studies, is quite useful for discovering new words. We show how to design features and how to improve not only the effect of each feature set but also the performance of the final model. We also describe experiments on a Vietnamese corpus to show the effectiveness of proposed model in comparison with previous approaches.

Towards POS tagging, motivated from the investigation of Chinese research (Low and Ng 2004) and the characteristics of Vietnamese, we present a new approach for Vietnamese POS tagging. In Vietnamese, every word consists of one or more morphemes which are separated by white spaces. Previous researches mostly extracted features based on units of words. In this paper, we present another way of feature design based on morpheme information (named 
morpheme-based features) which is more useful for Vietnamese POS tagging. In addition, we also prepared a POS-tagged corpus (approximately 8,000 sentences) to conduct experiments and contribute to Vietnamese language resources.

The remains of this paper are organized as follows. Firstly, we introduce briefly some background knowledge of related models in section 2. Secondly, in section 3 and 4 we analyze the proposed model for Vietnamese word segmentation and POS tagging. In each section, we will also describe experiments and discuss results. Then, in section 5, we discuss our model versus previous works and prove the efficiency of proposed feature selection. Finally, section 6 is the conclusion.

\section{Overview of related models}

\subsection{Dictionary-based model}

Two typical methods of dictionary-based model are longest matching and maximal matching.

The longest matching method scans all input sentence from left to right and selects the longest match existing in a given dictionary. This method will wrongly segment in many cases because of its drawbacks. For example, in the sentence in Figure 1, the original one will be incorrectly segmented as the first one. While, the correct segmentation is second one.

The maximal matching method first generate all possible segmentations for an input sentence and then select the one that contains fewest words. This method cannot determine the best segmented sentence in the cases that multiple segmented results have the same number of words. For example, the sentence (see Figure 2) has two candidates called $1^{\text {st }}$ and $2^{\text {nd }}$ word-segmented sentence. In cases like this, some other heuristic methods should be applied to determine the best one.

\begin{tabular}{|c|c|c|}
\hline 1 & Original sent. & Đó là cách đề truyền thông tin \\
\hline 2 & $1^{\text {st }}$ word-segmented sent. & Đó là cách đề truyền_thông tin \\
\hline 3 & $2^{\text {nd }}$ word-segmented sent. & Đó là cách đề truyền thông_tin \\
\hline
\end{tabular}

Fig. 1 An example of LM example.

\begin{tabular}{|c|c|c|}
\hline 1 & Original sent. & Học sinh học sinh học \\
\hline 2 & $1^{\text {st }}$ word-segmented sent. & Học_sinh học_sinh học \\
\hline 3 & $2^{\text {nd }}$ word-segmented sent. & Học_sinh học sinh_học \\
\hline
\end{tabular}

Fig. 2 An example of MM example. 


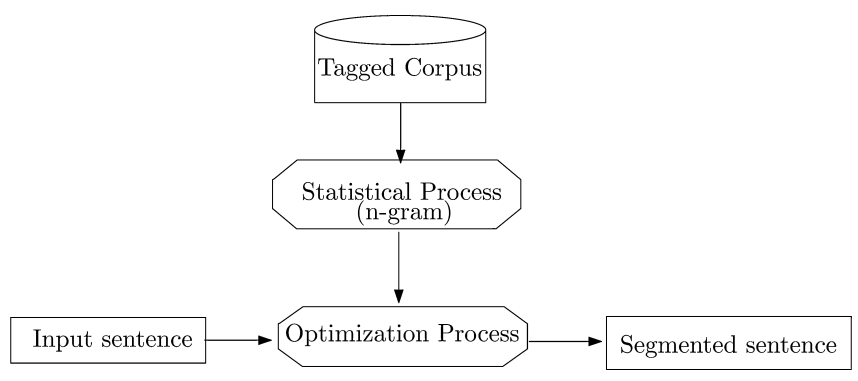

Fig. 3 Word segmentation using N-gram model.

\subsection{N-Gram based model}

An n-gram language model can express relatively well the relationship of the word's context. In this model, each word depends probabilistically on the n-1 preceding words.

$$
P(W)=P\left(w_{1} w_{2} \ldots w_{n}\right)=\prod_{i=1}^{n} P\left(w_{i} \mid w_{i-n+1} \ldots w_{i-1}\right) .
$$

Figure 3 illustrates a word segmentation system using N-gram model. In this model, a sequential list of $\mathrm{n}$ words and its frequency are determined. Basing on that statistic, with each input sentence, optimization process will select the solution which maximizes probability product $P(W)$ according to fomular 1 .

This method seems to be the main statistic one for identifying word boundary of new lexical units when there is no information from dictionaries as well as annotated corpus. The challenge is that we must find a good score to evaluate whether a given morpheme conjunction is a word or not. There are several methods to choose that score. For example, Maosong et al. (Maosong 1998) use mutual information and t-scores to identify word boundary in Chinese, and the reported results are very high even though challenged by other authors. For Vietnamese word segmentation, Le Ha (Ha 2003) used n-gram frequency to optimize the probability of given chunk with promising results.

\subsection{Named entity recognition model}

NER aims to classify each word in a text to one of predefined class names such as personal name, location name, organization name, date time, percentage, number and monetary value. An example of NER-labeled sentence is given in Figure 4.

Many machine learning methods have been applied to NER task, in which they mainly rely on linguistic features and context features to identify its own type. For example, Tri Tran Q. (Tri 


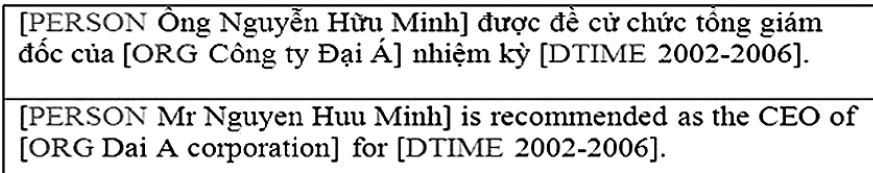

Fig. 4 An example of NER_labeled sentence.

et al. 2007) presented the results of an experiment in which a Support Vector Machine based NER model is applied to Vietnamese. J. Gao and et al. (Gao 2005) proposed a pragmatic mathematical framework in which segmenting Chinese known words and detecting unknown words of different types (i.e. morphologically derived words, factoids, named entities). In these works and many other researches like (Cam-Tu Nguyen 2007) also pointed out that NER task has a close relation to word segmentation.

\section{The proposed model for vietnamese word segmentation}

\subsection{Analyzing proposed models}

The performance of dictionary-based methods like Longest Matching and Maximum Matching mostly depends on the coverage of dictionaries. Unfortunately, there has not existed a complete dictionary that covers all entries of a specific language because out of vocabulary words (OOVs) appear commonly in documents. In statistics, Chinese SIGHAN's PK corpus has approximately $30 \%$ of OOV words (Gao 2005); Vietnamese corpus that we used to estimate our model has approximately 11.6\% OOV words (Cam-Tu Nguyen 2007). Even, in the case of existing an ideal dictionary, dictionary-based methods still cannot handle some ambiguous instances. The challenge is that how we can make use of these methods and overcome these difficulties.

We realize that, some OOVs are named entities and some others are factoid forms. Thus, in this paper we handle two related problems as following:

- The first type is Named Entity (including personal name, organization name, location name) and factoid forms (which are Date Time, Duration, Time, Percent and fraction, Money, Number, Measure, Email, Phone number, fax, WWW).

- The second type is new words that are not recognized as named entity or factoid forms. These new words are usually domain-specific, time-sensitive and culture-related terms.

To handle ambiguous instances, we use a heuristic method to choose the best segmentation according to its context. With regard to Named Entities and Factoid forms, we study methods 
used to recognize Named entities as well as Factoid forms correspondingly. Factoid forms can be easily captured by regular expressions, however, named entities are not recognized similarly. We had to research characteristics of Vietnamese named entities and models to recognize Vietnamese named entity such as (Tu et al. 2005) (Tri et al. 2007). Finally, in order to detect OOVs words of the second one, we utilize N-gram information to estimate how any morpheme conjunction may be a word. This information also helps to provide more knowledge in company with dictionary-based models.

As you can see in Figure 5, feature extractor will extract features for preprocessed documents by using information from related models including N-gram, NER, dictionary-based information and additional resources.

\subsection{Designing features for proposed model}

In the proposed model, we designed three feature sets which are presented in Table 1. Next, we discuss more these feature sets.

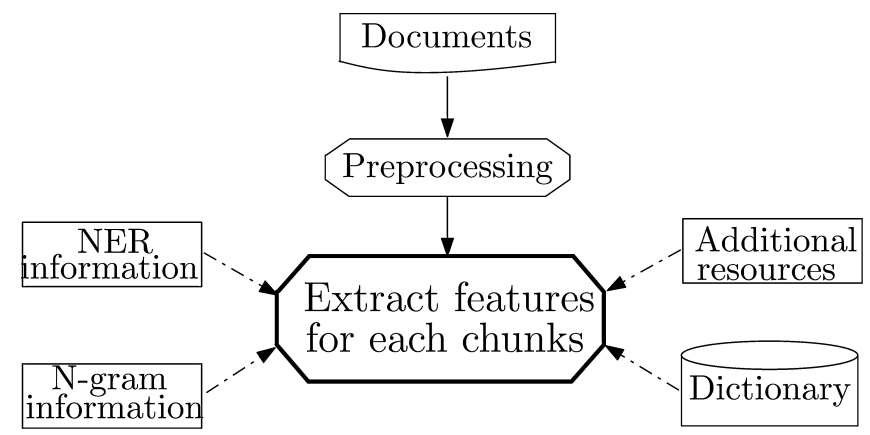

Fig. 5 Extract features from the information of other models.

Table 1 The proposed feature sets.

\begin{tabular}{|c|c|c|c|}
\hline No & Model & Type of Features & Detailed Features \\
\hline FS1 & Dict.-based word segmentation & Syllable Conjunctions & Each SC is a valid entry in dictionary? \\
\hline \multirow[t]{2}{*}{ FS2 } & \multirow[t]{2}{*}{ NER model } & $\begin{array}{l}\text { External Resource } \\
\text { - Dictionary } \\
\text { - Name List } \\
\text { - Location List }\end{array}$ & $\begin{array}{l}\text { Is each SC a valid Name? } \\
\text { In Location List? } \\
\text { Is-Regular-Expression }(0,0) \\
\text { Is-Initial-Capitalization }(0,0) \\
\text { Is_All_Capitalization }(0,0) \\
\text { Is_First_Observation }(0,0) \\
\text { Is_Marks }(0,0))\end{array}$ \\
\hline & & Factoid & Is_Regex \\
\hline FS3 & N-gram Model & $\mathrm{N}$-gram information & The log of probability (2-gram, 3-gram) \\
\hline
\end{tabular}




\subsubsection{Features from dictionary-based word segmentation model}

Unlike previous studies (Cam-Tu Nguyen 2007; Dien and Thuy 2006; Dien and Kiem 2001), instead of using both the left morphemes and the right morphemes of the current morpheme, we only use the information of the left morphemes. This suggestion is originated from the way of generating word candidates in Longest Matching method. With each morpheme conjunction (SC in abbreviation) we check if it exists as a valid entry in the dictionary or not. It is assumed that the maximum length of each Vietnamese word is 4 , accordingly we designed 4 features: $S C(-3,0), S C(-2,0), S C(-1,0), S C(0,0)$ which equals 1 if they are entries in dictionary and otherwise 0. These four features constitute the feature set 1 called FS1 (see Table 1).

For example, in the sentence (see Figure 6 ), if we extract features for the $4^{\text {th }}$ morpheme, we have the following features in accordance with FS1 (see Figure 6).

\subsubsection{Features from NER model}

Factoid forms are easily captured by regular expressions. Therefore, we had a feature called isRegex to capture factoid forms. We also cover 2 types of Vietnamese named entities which are personal names and location names.

A valid Vietnamese personal name usually has the first letter capitalized and conforms to following rule:

$A$ valid personal name $=A$ family name $+A$ middle name $+A$ given name Thus, to identify personal names, we rely on a Vietnamese personal name list which consists of about 21.000 names. From that list, we enumerated family name list, middle name list and given name list correspondingly. To give concrete features, with each morpheme conjunction above, we had a feature that equals:

- 1 if it conforms to the rule

- 0 if vice verse

To detect location names, we use a location name list of about 800 names. This will be the

\begin{tabular}{|c|c|c|}
\hline \multirow{2}{*}{ Sentence } & \multicolumn{2}{|c|}{$\begin{array}{c}\text { Thị trường điện thoại di động đang rất nóng } \\
\text { (The mobiphone market is very hot) }\end{array}$} \\
\hline Syllable & \multicolumn{2}{|c|}{ Feature Set } \\
\hline \multirow{3}{*}{ thoại } & $\mathrm{SC}(-3,0)$ & In_dictionary: 0 \\
\cline { 2 - 3 } & $\mathrm{SC}(-2,0)$ & In_dictionary: 0 \\
\cline { 2 - 3 } & $\mathrm{SC}(-1,0)$ & In_dictionary: 1 \\
\cline { 2 - 3 } & $\mathrm{SC}(0,0)$ & In_dictionary: 0 \\
\hline
\end{tabular}

Fig. 6 An example of FS1. 
foundation to design features aiding in recognizing them. In similar way, we had a feature that equals:

- 1 if each morpheme conjunction is an entry in location list

- 0 if vice verse

In common, the initial letter of the first morpheme in a sentence is usually capitalized, thus it is easy to mix with named entities. For this reason, we need one more feature named Is_First_Observation $(0,0)$. In addition, we also had a feature named Is_Mark to check if current morpheme is a mark. These are useful features to recognize exactly named entities in the text.

\subsubsection{Features from N-gram model}

As well known, the N-gram-based Word Segmentation model uses the probability of each $\mathrm{N}$-gram as a basic unit of information. To estimate the probabilities (parameters) of the Ngram model used in our model, we collected a corpus of approximately 14M-morpheme from http://vi.wikipedia.org/. This information will be employed in proposed model, in which bigram and tri-gram are used. Because these probabilities are too small, instead of using these values directly we get its $\log$ (see formula 2 ) and map into $[0,1]$ according to formula 3 .

$$
\begin{aligned}
& m i=\log (P(N-\operatorname{gram}))=\log (f)-\log (14000000) . \\
& I n f o(N-g r a m)=\left(1-\frac{|m i+| m a x_{-} N-g r a m||}{\left|m i n_{-} N-g r a m\right|}\right) .
\end{aligned}
$$

In statistic from our corpus ( $14 \mathrm{M}$-morpheme Wiki), we found that:

- $\mathrm{P}(2$-gram $):$ min_2-gram $\approx-41, \max \_2$-gram $\approx-8.00$

- $\mathrm{P}(3$-gram $):$ min_3-gram $\approx-41, \max \_3$-gram $\approx-10.00$

Thus, to map in $[0,1]$ we will replace values corresponding to its N-gram. For example, with the sentence "MobiFone dau tu hon 2 ti dong phat trien mang.", and the current morpheme $t$, we will extract two N-gram features as follow:

- $\operatorname{Info}(2$-gram $)=(1-\mid \log (\mathrm{P}($ "dau tu” $))+8.00 \mid / 41)=0.922$

- $\operatorname{Info}(3$-gram $)=(1-\mid \log (\mathrm{P}($ "MobiFone dau tu" $))+10.00 \mid / 41)=.700$

These features imply that the higher their values are, the higher the probability of these morpheme conjunctions forming a word becomes.

\subsection{Experimental results}

In the experiments, to utilize all the extracted features we use a maximum entropy model (MEM). MEM is one of promising methods proposed for natural language processing problems. Using MEM, Vietnamese word segmentation can be regarded as a classification problem 
in which each morpheme belongs to one of two labels named $B_{-} W$ (Begin_of_Word) and $I_{-} W$ (Inner_of_Word). An example of the input sentence and its output are presented in Figure 7.

The sentences in our corpus were collected from newspapers of multiple domains (economics, information technology, education, vehicle, sport, law, culture-Society) with the size of approximately 8,000 Vietnamese sentences. This corpus is divided into 5 folds and it is used to perform 5-fold cross validation test. In our experiment, we consider Longest Matching method as a baseline. Next, we report experiment results comparing to previous models and describe some experiments to examine the effect of each kind of features on the final model and the performance of the final model. This helps to find out which features are the most useful.

\subsubsection{Compare the effectiveness of FS1 and FS2 to previous approaches}

To estimate the effectiveness of these feature sets, we did some experiments to compare with previous approaches. As you can see in Table 2, if we used only left morphemes, we get better results in comparison with the results when using both left and right morphemes. In addition, when extracting featured based on NER, instead of checking each morpheme is in the family name list or the middle name list or the given name list (as in work (Cam-Tu Nguyen 2007)), we check whether each morpheme conjunction is a valid name or not. The results on Table 3 showed that our approach get better results. The reason is that in Vietnamese, first name, last name and middle name may be the same, so the approach in (Cam-Tu Nguyen 2007) will make confused.

\begin{tabular}{|c|c|c|c|c|c|c|}
\hline Thi & trường & chứng & khoán & đang & đi & xuống \\
\hline B_W & I_W & B_W & I_W & B_W & B_W & B_W \\
\hline \multicolumn{2}{|c|}{ The market } & \multicolumn{2}{|c|}{ stock } & being & go & down \\
\hline
\end{tabular}

Fig. 7 Example of segmented sentence.

Table 2 Result to estimate the importance of Dictionary-based features

\begin{tabular}{c|c|c|c|c}
\hline No & Features based on & Precision & Recall & F1 measure \\
\hline 1 & Left and right morphemes & 94.03 & 93.64 & $\mathbf{9 3 . 8 4}$ \\
2 & Only left morphemes & 94.95 & 94.2 & $\mathbf{9 4 . 5 8}$ \\
\hline
\end{tabular}

Table 3 Result to estimate the importance of NER-based features.

\begin{tabular}{c|c|c|c|c}
\hline No & Features based on & Precision & Recall & F1 measure \\
\hline 1 & Old & 94.92 & 94.22 & $\mathbf{9 4 . 5 7}$ \\
2 & Our approach & 95.15 & 94.43 & $\mathbf{9 4 . 7 9}$ \\
\hline
\end{tabular}




\subsubsection{Estimating the importance of each kind of feature sets}

To estimate how important each feature set is, we designed 2 types of experiments using Maximum Entropy Model. In the first type of experiment, we rejected each feature set in shifts and the results is shown in Table 4 . In the second one we only used each kind of feature set one after the other and the results is shown in Table 5. Finally, to estimate the power of proposed model, we performed word segmentation using all kinds of feature sets.

The results showed that dictionary-based feature set has the biggest effect: F1 is worst $(87.05 \%)$ if we did not use Dict.-based features; and if we only use each kind of feature set in turn, Dict.-based information give the best F1 (94.58\%). This can be explained because dictionaries play an important role in all high-performance word segmentation systems. The next is the effect of NER-based feature set (reject these feature set we get $93.55 \% \mathrm{~F} 1$ and use only them we get $91.32 \mathrm{~F} 1)$. The last is the effect of N-gram model.

The results also showed that if we added N-gram-based features to the model, the precision increases but the recall decreases. The reason is that some morpheme conjunctions was not true words but they appeared with high frequency in un-annotated corpus, therefore N-gram-based features got high values. Even in that case, we still got higher F1 measure.

As expected, MEM achieves the best result when using all kind of feature sets with $95.30 \%$ F1 measure. This result is higher than the result (94.23\% F1 measure) reported in (Cam-Tu Nguyen 2007) when testing on the same corpus.

Table 4 Experimental result of rejecting each feature set alternatively.

\begin{tabular}{c|c|c|c|c|c}
\hline No & Not use (Reject) & Method & Precision & Recall & F1 measure \\
\hline 1 & - & Longest Matching & 81.07 & 87.97 & $\mathbf{8 4 . 5 2}$ \\
2 & Dict.-based feature set & MEM & 96.99 & 77.1 & $\mathbf{8 7 . 0 5}$ \\
3 & NER-based feature set & MEM & 97.21 & 89.88 & $\mathbf{9 3 . 5 5}$ \\
4 & N-gram-based feature set & MEM & 95.15 & 94.43 & $\mathbf{9 4 . 7 9}$ \\
5 & & MEM & 96.71 & 93.89 & $\mathbf{9 5 . 3 0}$ \\
\hline
\end{tabular}

Table 5 Experimental result of using only each kind of feature set.

\begin{tabular}{c|c|c|c|c|c}
\hline No & Only use & Method & Precision & Recall & F1 measure \\
\hline 1 & - & Longest Matching & 81.07 & 87.97 & $\mathbf{8 4 . 5 2}$ \\
2 & Dict.-based feature set & MEM & 94.95 & 94.2 & $\mathbf{9 4 . 5 8}$ \\
3 & NER-based feature set & MEM & 90.89 & 91.74 & $\mathbf{9 1 . 3 2}$ \\
4 & N-gram-based feature set & MEM & 97.98 & 60.5 & $\mathbf{7 9 . 2 4}$ \\
5 & All & MEM & 96.71 & 93.89 & $\mathbf{9 5 . 3 0}$ \\
\hline
\end{tabular}




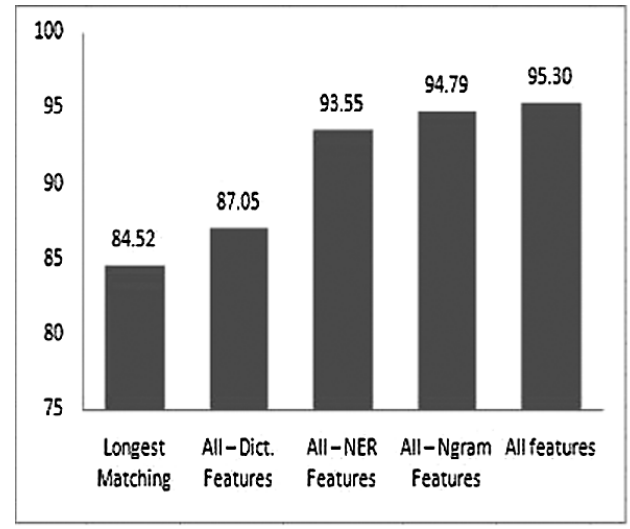

Rejecting each feature set in turn

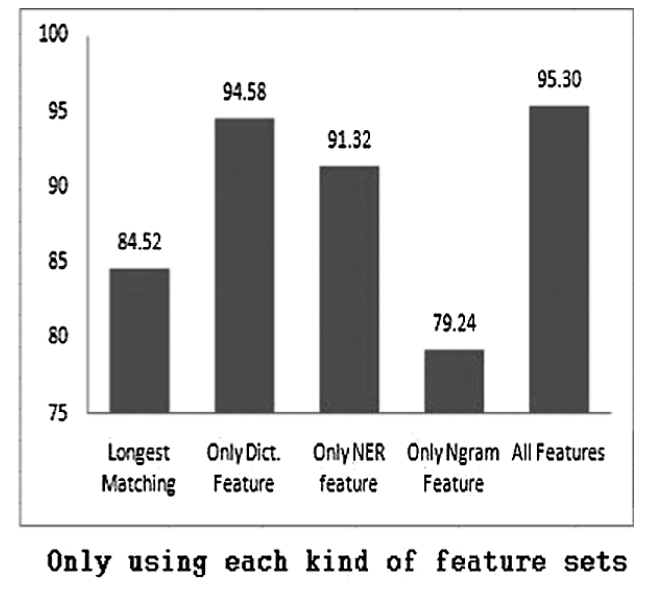

Fig. 8 The chart of F1 measure.

We also measured the rate of OOVs hit by the proposed model and saw that this model can correctly recognize $20 \%$ greater than Longest Matching method. We observed that these OOVs are usually high-frequency words besides named entities. Therefore, if data contains lots of high-frequency words then Ngram-based features will become more effective.

Figure 8 visualized F1 measure of 2 types of experiments.

\section{Proposed model for vietnamese POS tagging}

This section propose a new approach for Vietnamese POS tagging using MEM. We investigate another feature design named morpheme-based features. In Vietnamese, there is no standard corpus or public corpus. Therefore, in order to conduct experiments for POS tagging task we first build a Vietnamese POS-tagged corpus manually.

\subsection{Corpus Building}

In this study, we built a Vietnamese POS corpus based on word-segmented corpus from the result of paper (Cam-Tu Nguyen 2007). To do that, we performed the following tasks:

- Designed a Vietnamese POS tag set including 14 tags and $>10$ symbolic tags

- Built a tool to assign POS tag quickly

- Assigned POS tag for approximately 8,000 sentences belonging to multiple topics

Choosing a sufficient tag set plays an important role in POS tagging task. A large tag set will increase difficulties but a small one may not provide enough information to a specific purpose. 
Therefore, it is necessary to have a compromise between two factors which are able to do POS tagging and achieved information. With Vietnamese, designing a good tag set becomes more difficult because word class separation is still a controversial problem (Ban 2000) (Hoa 2004). According to Diep Quang Ban (Ban 2000), word class separation usually has three criteria which are general meaning, combining ability, and syntactic function. To assist in corpus building and some applications such as question answering, text summarization, we determined Vietnamese POS tag set at coarse-grained level with some modifications as listed in Table 6. After determining this POS tag set, we performed POS tagging over word segmented corpus with approximately 8,000 sentences. The corpus is now available for downloading at the web site http://vnlp.net/blog/?p=164.

\subsection{Designing feature sets for vietnamese POS tagging model}

Feature selection is one of the most crucial factors that affect directly the performance of a machine learning model. The better features we select, the higher the precision of POS tagging model we will obtain. When designing features, we comprehend typical features which are commonly used in other languages. Concurrently, we also add some more features which are suitable for Vietnamese characteristics. Because Vietnamese words consist of one or more morphemes, it is necessary to consider designing features at both word level and morpheme level.

\subsubsection{Word-based features}

To find out useful features for Vietnamese POS tagging, firstly we investigate the feature

Table 6 Vietnamese tag set.

\begin{tabular}{c|c|c}
\hline No & Tag Name & Describing \\
\hline 1 & NN & normal noun \\
2 & NC & classifier noun \\
3 & NP & proper noun \\
4 & VB & verb \\
5 & JJ & pronoun \\
6 & PP & adverb \\
7 & D & Determiner and cardinal number \\
8 & AD & preposition \\
9 & IN & Conjunction word \\
10 & CC & interjection \\
11 & UH & particle \\
12 & RB & idiom \\
13 & TN & unknown word \\
14 & X &,.$+=? .$. \\
$15++$ & symbols & \\
\hline
\end{tabular}


selection that was successfully applied for English (Ratnaparkhi 1997). In that research, they extracted features based on word information and its surrounding contexts. Similarly, for Vietnamese, we also extract corresponding word-based features. Features are described as follows:

- Words $W_{i}(i=-2,-1,0,1,2)$

- Word conjunctions with current word $W_{0}$ giving window size $=2$

- POS tag of the previous word $\operatorname{POS}\left(W_{-1}\right)$

- A pair of POS tags of 2 previous words $P O S\left(W_{-2}\right) \operatorname{POS}\left(W_{-1}\right)$

- Is current word punctuation?

- Is current word capitalized?

\subsubsection{Morpheme-based features}

Motivated from Chinese research (Low and Ng 2004) and Vietnamese characteristics, we investigated a different feature selection - called morpheme-based features. This means that we design features based on morpheme information. For more details, these features are shown as follows:

- Morphemes $M_{i}(i=-2,-1,0,1,2)$

- Morpheme conjunctions with current morpheme $M_{0}$ giving window size $=2$

- POS tag of the previous morpheme $\operatorname{POS}\left(M_{-1 W o}\right)$

- A pair of POS tags of 2 previous morphemes POS $\left(M_{-2 W o}\right) P O S\left(M_{-1 W o}\right)$

- Is current morpheme punctuation?

- Is current morpheme capitalized?

We notice that feature $P O S\left(M_{-1 W o}\right)$ refers to the POS tag of the previous morpheme before the current word. And $\operatorname{POS}\left(M_{-2 W o}\right) P O S\left(M_{-1 W o}\right)$ refers to the POS tags of two previous morphemes before the current word. For example, in the sentence "Ong duoc biet den la nha quan_ly tai_ba." (He is known as a talented manager.), when considering morpheme "ba", these two features get values VB and NC_VB (assumes that POS tag of " $n h a$ " is assigned NC and "quan_ly" is assigned VB).

\section{Testing Phase}

When applying morpheme-based features, to calculate the probability of a word assigned a POS tag t, we will get the product of the probability of its individual morpheme being assigned the same POS tag $t$. This constraint makes sure that morphemes of a word cannot be assigned different POS tags. After training the model, we do POS tagging for each sentence in the testing corpus. The assigning process is performed from left to right. At each time, k-best POS sequence is stored and used to be context for the next word. Given a sentence $w_{1}, \ldots, w_{n}$, a POS sequence 
candidate is determined formally in equation 4.

$$
P\left(a_{1} \ldots a_{n} \mid w_{1} \ldots w_{n}\right)=\prod_{i=1}^{n} P\left(a_{i} \mid b_{i}\right) .
$$

$b_{i}$ is the history for word $i$. Instead of multiplying, we got logarithm and BEAM SEARCH is presented as follows.

- Generate tags for word $w_{i}$, find out N-best tags assigning into $\mathrm{N}$ tag sequences called $S_{j}(\mathrm{j}$ $=1, \mathrm{~N})$

- For $\mathrm{i}=2$ to $\mathrm{n}$ ( $\mathrm{n}$ is the length of the sentence)

- For $\mathrm{j}=1$ to $\mathrm{N}$

* Generate tags for word wi given $S_{j}$ is previous tag sequence

* Concatenate this tag to the end of $S_{j}$

- From these tag sequences, find out N-best tag sequences named $S_{j}(\mathrm{j}=1, \ldots, \mathrm{N})$

- Return the best tag sequence $S_{1}$

In all experiments, to estimate the model we chose beam size $=3$.

\subsection{Experimental results of proposed POS tagging model}

\subsubsection{Experiment set up}

In accompany with built corpus (called $v n P O S$ ), we also conducted corresponding experiments based on another corpus named Viettrebank (VLNP 2009). This corpus consists of approximately 10.000 sentences of multiple topics. It was built based on a tag set of 17 POS tags - the result of national project VLSP.

With each corpus, we divided it into five folds, then we use cross validation to do estimation. In each time, we use $4 / 5$ to train the model and $1 / 5$ to test the model. In all experiments, we use an implementation of BLMVM algorithm (Benson et al. 2001).

\subsubsection{Experimental results}

The experimental results (presented in Table 7) indicated that when using word-based features, average precision $(\approx 86 \%)$ was remarkably lower in comparison with results researchers did for English, Japanese or Chinese. To find out more effective features for Vietnamese POS tagging, we also did experiments using morpheme-based features.

With Viettrebank corpus, we got $86.42 \%$ average precision if using word-based features and $89.87 \%$ average precision if using morpheme-based features; With vnPOS corpus, we got $85.57 \%$ average precision if using word-based features and $89.30 \%$ average precision if using morphemebased features. In MEM framework, we realized that using morpheme-based features always 
Table 7 The precision (\%) of Vietnamese POS tagging result

\begin{tabular}{c|c|c}
\hline Corpus & $\begin{array}{c}\text { ViettreBank } \\
\text { W-based \& M-based }\end{array}$ & $\begin{array}{c}\text { vnPOS } \\
\text { W-based \& M-based }\end{array}$ \\
\hline Fold 1 & $86.47-89.72$ & $85.17-88.63$ \\
Fold 2 & $86.73-89.93$ & $85.64-89.64$ \\
Fold 3 & $86.56-89.76$ & $85.51-89.26$ \\
Fold 4 & $86.24-90.07$ & $85.71-89.36$ \\
Fold 5 & $86.11-89.86$ & $85.81-89.63$ \\
\hline Average & $86.42-89.87$ & $85.57-89.30$ \\
\hline
\end{tabular}

give better average than word-based features (increased $3.73 \%$ on vnPOS corpus and $3.45 \%$ on ViettreBank corpus). It can be said that with Vietnamese POS tagging, morpheme-based features is more suitable than word-based features.

\section{Discussions and related works}

There are two architectures for POS tagging task in a real application. The first one is pipeline approach and the second one is integrating approach.

For the first one, the output of word segmentation is the input for POS tagging-pipeline. This is also the selected approach in this study. In this case the POS tagging task has to accept some errors of input as the result of word segmentation. Therefore, to fairly evaluate POS tagging, as other studies, we assume that POS tagging using a gold standard data of word segmentation.

For the second one, there are some models as presented in (Low and Ng 2004) (Shi and Wang 2007) for integrating word segmentation and POS tagging concurrently. In order to survey, we have already investigated some models for integrating word segmentation and POS tagging tasks. In the integrated model, these two tasks are considered as a classification problem in which each morpheme will be tagged with two labels, one for word segmentation and one for POS tagging. In the experiments, we got $93.42 \% \mathrm{~F} 1$ for word segmentation and $86.40 \% \mathrm{~F} 1$ for POS tagging task. These obtained results are not good in comparison with performing them separately. Therefore in this paper we just only present the pipeline approach. In the following, we will compare our best model to previous models for each task.

\subsection{Related word segmentation model}

There are several models proposed for Vietnamese word segmentation. Le An Ha (Ha 2003) built 10M-morpheme raw corpus and used n-gram information to optimize sum of segmentation 
probabilities. The experimental results were not high but proved that N-gram is useful if we make use of its advantage to combine with other information. Cam-Tu et al. (Cam-Tu Nguyen 2007) have investigated the use of CRF and SVM models for solving this task. In other work, Dinh Dien et al. (Dien and Thuy 2006) have used maximum entropy framework to train on annotated corpora. In that work, they separated unknown word recognition and known word segmentation as two independent processes. However, it is believed that the identification of unknown words should not be separated from word segmentation. One typical example of such approach is Gao and et al. (Gao 2005).

Unlike the approach in (Dien and Thuy 2006), we solve unknown word identification and known word segmentation concurrently. This idea is like the work in (Cam-Tu Nguyen 2007) and it is believed to give a better result (Gao 2005). Previous works stated that the features impact to the performance significantly. This means that feature selection decides the performance of the final model. For this reason, we investigate the information that has large effects on the word segmentation task. We designed 3 feature sets which are different from feature sets of previous approaches (Dien and Thuy 2006) (Cam-Tu Nguyen 2007). With the same corpus, our proposed model give better performance in comparison with research in (Cam-Tu Nguyen 2007). We got 95.30\% F1 measure (1.07\% higher). To compare with research of (Dinh Dien and Vu Thuy 2006), we also tested on the corpus of Vietnamese Lexicography Center (www.vietlex.com.vn), and the results showed that our model got higher F1 (we got $94.76 \%$ in comparison to $94.44 \%$ ). Experimental results demonstrated that our approach of feature selection really works. We add one more type of features to the model which is N-gram-based featuers. This is completely new features compared to previous approaches. The result of the experiments using each kind of feature sets and using all feature sets above demonstrated that we got higher results than previous ones. This means that these types of feature sets bring more information to MEM model when it is applied to word segmentation task.

\subsection{Related POS tagging model}

Currently, there are some studies for Vietnamese POS tagging obtaining noticeable results. For example, Nguyen Thi Minh Huyen and et al. built a corpus and a probabilistic tagger named vnQTAG (Huyen 2003). In that research, they modified QTAG software to adapt it to Vietnamese texts as well as allow using lexicon dictionary containing POS information. The idea is determining probability distribution over the space combining word sequences and corresponding POS sequences. Minh Nghiem and Dien Dinh (Minh and Dien 2008) proposed a robust method for POS tagging on Vietnamese documents by using a wide variety of features, including language 
specific features. They used SVM method to perform POS tagging. All these researches usually designed features based on word-based information. In this study, we not only took full advantages of above results but also investigated another feature design based on word composition. We presented another way of feature design based on morpheme information. And the experimental results on two POS-tagged corpora proved that morpheme-based features are better by far.

\section{Conclusion and future works}

In this paper, we presented a novel approach to Vietnamese word segmentation in which we tried to use multiple knowledge resources from distinct models. We integrated the information from models including N-gram, NER, and dictionary-based method into a maximum entropy model to improve the accuracy of the proposal model. Basing on the investigation the rich information from these model and previous approaches (Cam-Tu Nguyen 2007), (Dien and Thuy 2006), we showed out the best way of extracting feature sets. The experimental results demonstrated that the effect of each kind of knowledge resource on the performance of the word segmentation model is different from each other. Particularly, dictionary-based features are the most important features, the next are the effect of NER-based features, and the third is the effect of N-gram features. The experimental results also showed that proposed model has the power of the state-of-the-art models.

In this paper, we also presented a new approach for Vietnamese POS tagging task and tried to find out useful feature sets for this problem. After investigating previous researches, we proposed a new feature design which is called morpheme-based features. To find out which features are more useful, we did experiments based on MEM model using two corpora. The experimental results showed that morpheme-based features are remarkably better than word-based features for Vietnamese POS tagging task. In the best case, we achieved the precision of $89.64 \%$ using morpheme-based features on VietTreeBank corpus.

In addition, to contribute into Vietnamese language resources that are in building process, we also prepared a Vietnamese POS-tagged corpus. This corpus includes approximately 8,000 Vietnamese sentences collected from electronic newspapers of multiple domains. The corpus is available for downloading at the web site http://vnlp.net/blog/?p=164. 


\section{Acknowledgement}

This work was supported in part by the MoST-203906 Project and scientific research project QG.07.25.

\section{Reference}

Cam-Tu Nguyen, Trung-Kien Nguyen, Xuan-Hieu Phan, Le-Minh Nguyen, and Quang-Thuy Ha (2007). "Vietnamese Word Segmentation with CRFs and SVMs: An Investigation" In Proceeding of the 20th Pacific Asia Conference on Language, Information and Computation (PACLIC20), Wuhan, China, pp. 215-222.

Diep Quang Ban and Hoang Ban. (2000). "Vietnamese Grammar" In Book published by Education Publisher, Hanoi.

Dinh Dien and Vu Thuy. (2006). "A Maximum Entropy Approach for Vietnamese Word Segmentation" In Proceedings of 4 th IEEE International Conference on Computer ScienceResearch, Innovation and Vision of the Future, HoChiMinh City, Vietnam, pp. 12-16.

Dien, D., Kiem, H., and Toan N. V. (2001). "Vietnamese Word Segmentation" In The 6th Natural Language Processing Pacific Rim Symposium, pp. 749-756. Tokyo, Japan.

Fahim Muhammad Hasan, Naushad UzZaman, and Mumit Khan (2007). "Comparison of unigram, bigram, hmm and brill's pos tagging approaches for some south asian languages" In Comparison of unigram, bigram, hmm and brill's pos tagging approaches for some south asian languages.

Gao, J. F., Li, M., Wu A., and Huang, C. N. (2005). "Chinese word segmentation and Named Entity Recognition: A Pragmatic Approach" In Computational Linguistics. MIT Press.

Hwee Tou Ng and Jin Kiat Low (2004). "Chinese part-of-speech tagging: One-at-a-time or all-at-once? word-based or character-based?" In Proceedings of EMNLP, pp. 277-284.

James Mayfield, Paul Mc Namee, and Chiristine Piatko (2003). "Named Entity Recognition using Hundreds of Thousands of Feature" In Proceeding of CoNLL-2003, pp. 184-187. Edmonton Canada.

Church, K., Hanks, P., Gale, W., and Hindle, D. (1991). "Using Statistics in Lexical Analysis" In U. Zernik Lexical Acquisition: Using On-line Resources to Build a Lexicon, Lawrence Erlbaum Associates.

Le An Ha (2003). "A method for word segmentation in Vietnamese" In Proceedings of Corpus Linguistics. Lancaster, UK. 
Maosong Sun, Dayang Shen, and Benjamin K. Tsou (1998). "Chinese word segmentation without using lexicon and hand-crafted training data" In Proceeding. of COLING-ACL, pp. 12651271.

Mekanavin, S., Charenpornsawat, P., and Kijsirikul, B. (1997). "Feature-based Thai Words Segmentation" In Proceedings of the Natural Language Processing Pacific Rim Symposium, pp. 41-48, Phuket, Thailand.

Nguyen Cam Tu, Tran Thi Oanh, Phan Xuan Hieu, and Ha Quang Thuy (2005). "Named Entity Recognition in Vietnamese Free-text and Web Documents Using Conditional Random Fields" In The 8th conference on some Selection problems of Information Technology and Telecommunication, Hai Phong, Vietnam.

Nguyen Chi Hoa. (2004). "Practical Vietnamese Grammar" In Book published by Vietname National University Publisher, Hanoi.

Nghiem, M. Dien Dinh Nguyen, M. (2008). "Improving Vietnamese POS tagging by integrating a rich feature set and Support Vector Machines" In Proceedings of Research, Innovation and Vision for the Future, 2008. RIVF, pp. 128-133.

Nguyen Thi Minh Huyen, Vu Xuan Luong, and Le Hong Phuong (2003). "Probabilistic POS tagging QTAG for Vietnamese Text" In Proceedings of ICT.rda'03 Conference, Hanoi, Vietnam. Poowarawan, Y. (1986). "Conditional random fields: Dictionary-based Thai Syllable Separation" In Proceedings of the Ninth Electronics Engineering Conference.

Wong, P. and Chan C. (1996). "Chinese Word Segmentation based on Maximum Matching and Word Binding Force" In Proceedings of Coling 96, pp. 200-203.

Ratnaparkhi A. (1997). "A simple introduction to maximum entropy models for natural language processing" In Technical Report 97-08, Institute for Research in Cognitive Science, University of Pennsylvania.

Steven J. Benson and Jorge J. More (2001). "A Limited-Memory Variable-Metric Method for Bound-Constrained Minimization" Preprint ANL/MCS-P909-0901.

Tri Tran Q., Thao Pham T. X., Hung Ngo Q., Dien Dinh, and Nigel Collier (2007). "Named entity recognition in Vietnamese documents" In Progress in Informatics, pp. 5-13.

Yanxin Shi and Mengqiu Wang (2007). "A dual-layer CRF based joint decoding method for cascade segmentation and labelling tasks" In Proceedings of the IJCAI Conference, Hyderabad, India.

VLNP (2009). "VNLP" http://www.jaist.ac.jp/ bao/VLSP-text/. 
Master Oanh Thi Tran: is now a lecturer of Department of Information Systems, College of Technology, VNU. She got her Bachelor degree of IT in 2006 and Master degree of IT in 2009 at COLTECH, VNUH. Her major researches are Natural Language Processing and Data Mining.

Dr. Anh-Cuong Le: achieved B.S. and M.S.degrees of Information Technology from University of Sciences, Vietnam National University of Hanoi (VNU) in 1998 and 2001 respectively. He received his $\mathrm{PhD}$ degree in the School of Information Science, Japan Advanced Institute of Science and Technology (JAIST), in 2007. He is now a lecturer at Department of Computer Science, Faculty of Information Technology, College of Technology, VNU. His main research interests include natural language processing and statistical machine learning.

Assoc. Prof. Quang-Thuy Ha: received his BS degree in Computation and Mathematics from Hanoi University of Sciences (HUS) in 1978 and his $\mathrm{PhD}$ degree in Information Technology in 1997 from HUS, Vietnam National University, Hanoi (VNU). He is currently an associate professor in information systems and the head of the Knowledge Engineering Laboratory at College of Technology (COLTECH), VNU. His main research interests include rough sets, data mining, and information retrieval.

(Received May 16, 2009)

(Revised December 9, 2009)

(Accepted January 10, 2010) 